\title{
Introduction: scaling biodiversity - what is the problem?
}

\author{
DAVID STORCH \\ Charles University, Prague, The Santa Fe Institute \\ PABLO A. MARQUET \\ Pontificia Universidad Católica de Chile, CASEB, IEB, The Santa Fe Institute \\ JAMES H. BROWN \\ University of New Mexico, The Santa Fe Institute
}

Biological diversity is the most fascinating phenomenon on the Earth. Biologists, amazed by the splendid variety of life, spent several centuries collecting, describing, and classifying living things. We are still engaged in this endeavor. Some groups, such as birds, mammals, molluscs, and vascular plants, have received most of the attention, while others, such as mites, nematodes, fungi, and prokaryotes, remain very poorly known. Moreover, we are still only beginning to understand in depth the processes that generate and maintain the global biodiversity. Part of our ignorance comes from the complexity of observed biodiversity patterns and of the processes that have produced them. These range from evolutionary events that occurred millions of years ago to contemporary interactions between individual organisms and their environments, from biogeographic processes that play out on the scale of continents and oceans to local interactions that can occur on miniscule spatial scales. Part is simply due to the fact that the diversity of life is determined by a multitude of processes which are unique for each taxon and each environment: each kind of organism has unique features of structure and function, which are due to evolutionary constraints and which affect its strategies for survival and reproduction, each type of habitat has its unique abiotic conditions and biotic composition and its own dynamics, and each land mass and body of water has its own geological, climatic, and organic history. Searching for universal laws might seem to be a hopeless task.

There are, however, general, perhaps universal, patterns of biodiversity, suggesting that they might be due to equally general underlying processes. Biological diversity increases with the area sampled, decreases from the equator towards the poles, and is generally high in hot and humid places. Species richness tends to increase with total abundances of individuals and is promoted by 
Cambridge University Press

978-0-521-87602-5 - Scaling Biodiversity

Edited by David Storch, Pablo A. Marquet and James H. Brown

Excerpt

More information

the turnover in species composition of local communities, which, in turn, is affected by habitat heterogeneity and spatial aggregation of individuals. Also, although, or perhaps because, biodiversity is scale dependent, species richness of local ecological communities is always related to the richness of the larger surrounding biogeographic regions. Many potential explanations, some of them mutually exclusive, some not, have been advanced to explain these patterns (see e.g. Gaston \& Blackburn, 2000, and Blackburn \& Gaston, 2003, for reviews). Discerning between competing explanations requires careful formulation and quantitative testing of formal models relating pattern to processes (Storch \& Gaston, 2004).

The study of biodiversity is therefore a sophisticated quantitative modern science. Similarly as in other branches of science, it is necessary to discover and quantify those properties of systems that remain relatively invariant and stable regardless of the system-specific details and intricacies, and to develop formal models that capture the general features of system structure and behavior (Maurer, 1999). Such an approach has been very successful in disciplines such as statistical physics and cosmology, and is best exemplified by the theory and methodology of scaling. Scaling, in its broadest sense, is the effort to discover and explain how some state variable or dynamic parameter changes with some other variable.

Scaling in ecology is perhaps best developed in the context of spatial scaling, i.e. changes in observed patterns with the spatial scale of observation. Ecologists have long been aware that different patterns are apparent and different processes are operating on different spatial scales (e.g. Rahbek \& Graves, 2001; Whittaker, Willis \& Field, 2001; Rahbek, 2005). Only recently, however, have ecologists and biogeographers been able to reveal quantitative rules that describe how the patterns change across scales. This is an important first step toward a true scaling theory that would use models based on first principles to accurately predict such empirical scaling phenomena. Recent progress toward such quantitative treatment of biodiversity based on principles of scaling is the topic of this book.

The chapters in this volume are the written versions of talks presented at a workshop "Scaling Biodiversity", which was held in Prague, Czech Republic, on 19-22 October 2004. The workshop was cosponsored by the Santa Fe Institute and the Center for Theoretical Study, Charles University in Prague. It brought together an eclectic mix of scientists interested in biodiversity and scaling theory. These ranged from empirically oriented ecologists and biogeographers to mathematical biologists and theoretical physicists, and from graduate students and postdocs to eminent senior scientists. The lively interchange of data and ideas by individuals with very different backgrounds, approaches, and methodologies made for a memorable conference. Most participants agreed that the conference substantially broadened their own limited perspectives on 
biodiversity. It highlighted the many contributions that scaling approaches are now making to the emerging understanding of biodiversity patterns and processes.

The title of this volume thus refers to quantitative approaches to the patterns of biodiversity and the processes that generate them. We start, in the first part, with spatial scaling of species richness and its relationship to the spatial distribution and abundance of individual species. The second part considers other quantitative patterns, such as phylogenetic diversity and species spatial turnover. The third part tackles the role of energy availability, which appears to be a major driver of spatial biodiversity patterns, including the well-known latitudinal biodiversity gradient. The final part is devoted to more synthetic views on processes responsible for scaling phenomena and future perspectives on biodiversity scaling.

\section{Part I. Spatial scaling of species richness and distribution}

The notion that species richness depends on scale of observation is old, dating at least to the beginning of the twentieth century (Arrhenius, 1921; Gleason, 1922). The fact that the number of species on average increases with the area over which the species are counted is obvious, but the exact form of the species-area relationship is not. Empirical species-area relationships can take many forms depending on the spatial scale of study, taxon, environmental and geographical setting, and role of ecological and evolutionary processes (Rosenzweig, 1995). Nevertheless, there are also regularities. The species-area relationship tends to be triphasic - increasing rapidly at small spatial scales, then more slowly and approximately as a power law at intermediate scales, and then increasing rapidly again at the largest spatial scales. The overall shape of the species-area relationship is driven by the interplay between sampling effects, spatial population processes, and species turnover in response to habitat heterogeneity (Storch, Šizling \& Gaston, 2003). Whereas the effects of sampling and spatial population processes have been studied comprehensively, and their influences on species-area relationships have been analyzed (e.g. Hanski \& Gyllenberg, 1997; Hubbell, 2001), the effects of species-specific requirements in relation to spatially varying habitat structure have been largely neglected. Mike Palmer (Chapter 2) shows that the structure of habitat mosaics - i.e. along a gradient from fine-grained to coarse-grained - predictably affects the shape of the species-area relationship. Moreover, differences among landscapes in how habitat grain changes with spatial scale give testable predictions of how species richness scales with area.

Proximately, the shape of the species-area relationship is given by the spatial structure of the distribution of individual species: if all species had 
Cambridge University Press

978-0-521-87602-5 - Scaling Biodiversity

Edited by David Storch, Pablo A. Marquet and James H. Brown

Excerpt

More information

homogeneous distribution and occurred everywhere, species richness would not increase with area, and the more clumped the distributions of the species, the steeper the slope of the species-area relationship (He \& Legendre, 2002). Spatial distributions of individuals are almost never random (i.e. Poisson-like) nor simply clumped in large clumps without any internal structure, but rather they tend to be aggregated on all scales of resolution. This has led to the notion that species spatial distribution is fractal - i.e. self-similar - with quantitatively similar patterns of aggregation occurring on every spatial scale (Kunin, 1998), and that fractal species distributions are responsible for observed power-law species-area relationships (Harte, Kinzig \& Green, 1999; Šizling \& Storch, 2004).

But is spatial distribution of most species really fractal? And how do deviations from fractality affect the increase of species richness with increasing area? Fangliang He and Rick Condit (Chapter 3) show that although the increase of species relative occupancy with spatial scale can be often well approximated by a power law, indicating fractality in spatial distribution, a slightly different model fits even better. This suggests that better tools for analyzing and predicting spatial distribution of individuals, and consequent scaling of species richness, are needed. Jack Lennon and his colleagues (Chapter 4) used an original analytical technique to reveal fractality in the large-scale distributions of South African birds, finding that whereas spatial distributions of some species cannot be distinguished from true fractals, those of other species - especially the more abundant ones - deviate significantly from fractality, revealing less aggregated distributions at fine scales. Together, these two chapters question the generality and usefulness of strictly fractal approaches to species distributions and consequently to biodiversity scaling patterns.

By contrast, fractal patterns represent at least very good first approximations of the geometry of species spatial distributions; the distributions are definitely much closer to fractal than homogeneous or random. Are there biological reasons why the structure of species distribution should be self-similar? Arnošt Šizling and David Storch (Chapter 5) show that a distribution which is effectively indistinguishable from fractal can emerge by random multilevel aggregation driven by a hierarchical distribution of habitat patches, randomly nested within more broadly defined habitat types. This simple null model predicts well not only the observed scale-dependence of species occupancy patterns, but also the observed shapes of species-area relationships, and even the distribution of species abundances. The HEAP model of John Harte (Chapter 6) is similar in many of its tenets and makes similar predictions. This model also assumes random spatial aggregation of individuals on multiple scales, although the aggregation process is based on purely statistical principles rather than defined biological mechanisms (see Harte et al., 2005). However, Harte's chapter shows how the HEAP model can be interpreted in terms of random population growth-extinction processes, and can provide invaluable 
Cambridge University Press

978-0-521-87602-5 - Scaling Biodiversity

Edited by David Storch, Pablo A. Marquet and James H. Brown

Excerpt

More information

insights into how the spatial scaling of species abundance, distribution, and diversity are all related to each other.

\section{Part II. Alternative measures of biodiversity: taxonomy, phylogeny, and turnover}

The number of species co-occurring within an area is only one aspect of biological diversity. Different patterns can be revealed by measuring phylogenetic relationships or similarity and dissimilarity in taxonomic composition between communities. Careful consideration of such alternative measures is especially valuable whenever the concept of species or the definition of taxonomic units seems to be vague or unclear. This is especially true in the case of the hotly discussed issue of microbial biodiversity patterns. Some authors (e.g. Fenchel \& Finlay, 2004) claim that macroecological patterns in prokaryotes and unicellular eukaryotes are very different from those typically observed in large, multicellular plants and animals. They suggest that microbes are distributed relatively homogeneously across the Earth's surface. The idea is that microbes have phenomenal capacities for dispersal so they eventually colonize and occur everywhere that suitable conditions occur. The result is that similar environments have similar microbial species composition, regardless of their current spatial separation and evolutionary histories. However, as shown by Jessica Green and Brendan Bohannan (Chapter 7), this may be an artifact due to taxonomic resolution. New molecular genetic tools are revealing that a single morphologically recognizable microbial "species" can contain diverse genetically distinct populations, comparable in some cases to much higher taxonomic categories (genera, families, and even orders) of multicellular organisms. Therefore, as new tools reveal the full extent of diversity patterns in microbes, it will be important to incorporate genetic patterns and phylogenetic thinking to elucidate the processes underlying these patterns. In a similar vein, Jérôme Chave and his colleagues (Chapter 8) provide an example of how much additional information can be obtained by incorporating phylogenetic information into a quantitative study of forest biodiversity. Indeed, two hectares of forest with the same number of species can have very different diversity levels in terms of phylogenetic disparity between individuals. This means that the history of speciation and extinction, lineage diversification processes, and biogeographic events can leave important signatures in contemporary biodiversity.

Not only local species richness, but also species composition of communities change over space, especially in local ecological or larger-scale geographic gradients of environmental change. Spatial turnover of species contributes substantially to macroecological biodiversity patterns, because different regions can have very different levels of turnover between local communities. A major problem is how to measure turnover, which may depend on absolute levels of species richness as well as the spatial scale (grain size) of measurement 
Cambridge University Press

978-0-521-87602-5 - Scaling Biodiversity

Edited by David Storch, Pablo A. Marquet and James H. Brown

Excerpt

More information

(Koleff, Gaston \& Lennon, 2003). Tim Keitt (Chapter 9) proposes an efficient way of how to measure turnover simultaneously on a continuous range of spatial scales, showing how it depends on both scale and spatial location.

Since turnover in species composition between two sites depends on distance, habitat differences, and dispersal abilities of the organisms, it might seem that only few if any generalizations would be possible. However, Kevin Gaston and colleagues (Chapter 10) show that although various measures of turnover capture different features of the phenomenon, there are still some regularities. Similarity in species composition almost always decreases with distance and, when properly measured, it increases with local species richness and mean species occupancy. Such regularities by themselves cannot distinguish the roles of habitat heterogeneity and limited dispersal in generating turnover in species composition between distant sites; both niche-assembled and dispersalassembled species assemblages produce the same patterns. Nevertheless, observed regularities reveal the close connection between spatial patterns of species turnover and scaling patterns of species occupancy, abundance, and diversity.

\section{Part III. Scaling of biological diversity with energy and the latitudinal biodiversity gradient}

Diversity is not equally distributed across the Earth surface; some places are much richer than others. The most notable pattern is the latitudinal gradient of diversity, i.e. the decrease in species richness from the tropics towards the poles (Gaston, 2000; Willig, Kaufman \& Stevens, 2003). Andrew Clarke (Chapter 11) suggests that this pattern probably has several causes, ranging from contemporary climate to historical climatic and geological events. Moreover, the pattern is not absolutely universal. Some taxa are actually more diverse at high latitudes and altitudes. Pavel Kindlmann and colleagues (Chapter 12) supply evidence to suggest that these reverse gradients may be caused by negative relationships between the diversity of these exceptional organisms and of the more typical organisms that generate the "normal" gradients prevalent in most other taxa. Regardless of the possible multiple causality of the latitudinal gradient and some deviations from the common trend, however, there is a very strong and general relationship between biological diversity and climate (Hawkins et al., 2003). David Currie (Chapter 13) shows that terrestrial animal and plant diversity scales with key variables of temperature and humidity in essentially the same way throughout the world, so he infers that local ecological conditions constrain diversity so strongly that regional historical effects are relatively unimportant.

Temperature and moisture together largely control productivity, and therefore the quantity of resources available to a given taxon (but see Currie et al., 2004). In addition, however, temperature, through its effect on metabolic rate, 
could affect species richness through other mechanisms, such as rates of speciation, extinction, and ecological interaction (Allen, Brown \& Gillooly, 2002; Brown et al., 2004). Although Currie claims that species richness does not scale with temperature as predicted by metabolic theory, testing the theory is not straightforward. Andrew Allen and coauthors (Chapter 14) show that the deeper development and understanding of metabolic theory leads to slightly different predictions: namely the effect of temperature can be assessed only by comparing communities that contain approximately similar numbers of individuals and are not limited by water availability. This implies that species richness may be affected by the effect of temperature both on productivity through its influence on resource availability and on rates of ecological and evolutionary processes through its influence on metabolic rate. The current version of "metabolic theory" suggests how metabolic rate affects rates of both evolutionary and ecological processes, so its predictions about biodiversity are necessarily quite complex. As far as we know, however, it is the only theory providing quantitative predictions of scaling of species richness with environmental variables.

By contrast, some quantitative patterns relating species richness and energy availability can be derived from the knowledge of scaling of species richness with space. High environmental productivity leading to high species richness is often associated with higher probability of occurrence of individual species and consequently higher species occupancies (Bonn, Storch \& Gaston, 2004). This in turn leads to lower species turnover and shallower slopes of the species-area relationships in more productive areas and slower increase of richness with productivity within larger areas (Storch et al., 2005). As shown by David Storch, Arnošt Šizling and Kevin Gaston (Chapter 15), the assumption that the probability of species occurrence scales with both area and productivity is appropriate for realistically predicting both species-area and species-energy relationships, as well as of the interaction between them.

\section{Part IV. Processes, perspectives, and syntheses}

Many scaling rules mentioned above are based on quite simple geometric considerations and assumptions of static environmental constraints. However, all the patterns are in fact consequences of complex dynamical spatiotemporal processes, and these processes themselves reveal scaling laws. Species richness, for instance, increases predictably not only with spatial scale, but also with temporal window of observation (Preston, 1960), as discussed by Ethan White in his overview of this pattern (Chapter 16). Similarly as in the case of the interaction between the species-energy and species-area relationships, there is a negative interaction between species-area and species-time relationships: species richness increases more slowly with time in large areas. Does such apparent regularity in observed spatiotemporal patterns indicate some universal processes underlying them? What are the relevant processes? 
Cambridge University Press

978-0-521-87602-5 - Scaling Biodiversity

Edited by David Storch, Pablo A. Marquet and James H. Brown

Excerpt

More information

A hotly debated approach assumes that most essential processes can be modeled by treating all species as ecologically equivalent, with the result that community structure is then determined largely by stochastic events of birth, death, and local dispersal (Hubbell, 2001). Luís Borda-de-Água, Stephen Hubbell and Fangliang He (Chapter 17) used the so-called multifractal approach to show that models which assume such neutral dynamics and use realistic dispersal kernels predict scaling patterns of diversity and distribution very similar to those observed in tropical forests. Tomáš Herben (Chapter 18) applies the neutral approach to successfully predict patterns in species invasions, including relationships between richness of native and alien species, and higher susceptibility of islands than mainlands to invasion. These "neutral" models that generate diversity by assuming that all coexisting species share some very general features contrast markedly with traditional "niche models" that generate diversity based explicitly on differences among coexisting species.

Interesting patterns can also emerge when considering just one population of a species not interacting with other species, but constrained by local density dependence, dispersal, and/or habitat patchiness. William Kunin (Chapter 19) demonstrates how spatial structure of populations affects processes (namely local population extinction) that in turn affect population persistence and spatial structure. Beáta Oborny and colleagues (Chapter 20) show how local density dependence and dispersal limitation can lead to a "universal" spatiotemporal scaling behavior, which is independent of the many system-specific details. Indeed, population dynamics in space and time can lead to such phenomena as "critical states" and "scale invariance", which crucially affect population persistence, and which have been observed in complex physical systems. Pablo Marquet and colleagues (Chapter 21) develop similar themes, discussing the extent to which observed population abundances and their fluctuations reveal signs of universal scaling behaviors. As seen in several chapters, many population and community patterns can be approximated by power laws (see also Keitt \& Stanley, 1998; Keitt et al., 2002), again suggesting that some universal principles generate uniformity that lurks behind the observed variability.

\section{Concluding remarks}

We believe that this book captures the current diversity of the science of biodiversity. There is excitement and ferment, a heady variety of conceptual approaches, analytical techniques, and mathematical models. The chapters show how much progress has been made in just the last few years. Indeed, the depth of thinking, the number of environmental and historical factors being considered, and the sophistication of mathematical models and statistical analyses have all increased enormously. Many but by no means all chapters and their authors optimistically suggest that there are some general law-like patterns and 
processes underlying all of the many intricate details: the differences among taxa, environments, and historically and geographically isolated regions.

It is also clear, however, that any such general laws remain elusive. Many of the patterns may seem universal if analyzed in the "right" way, but the neutral and/or more deterministic ecological and evolutionary processes that have generated those patterns remain poorly understood. The chapters in this book amply illustrate the unfinished state of the science. Collectively, the chapters highlight the divergent approaches, contradictory assumptions, predictions, and interpretations, and still unanswered questions. Models that make very different assumptions are able to generate predictions that are very similar to each other and to observed empirical patterns. Alternative hypotheses, which may not necessarily be mutually exclusive, can be difficult to distinguish. There is obviously much unfinished business.

Nevertheless, it is equally obvious that progress is being made and the perspective of scaling is playing a major role. This perspective - the analysis of data and the development of models to understand how biodiversity varies across space, time, environmental conditions, and historical contingencies - has much more to contribute. We hope that some readers will be challenged to address the unresolved issues. And if they do so, we hope that they will find the information in this book to be useful, especially the emphasis on building and evaluating models that explore relationships between the patterns and processes, among the multiple variables and mechanisms, and across the disparate scales of space and time that characterize the enormous diversity of life on Earth.

Neither the workshop nor this book would have been possible without the help of many people. We thank the Santa Fe Institute (SFI) and the Center for Theoretical Study, Charles University (namely its research program MSM0021620845), for their generous support for the workshop and the preparation of this book. We are especially grateful to Barbora Svatá for substantial help in organizing the workshop as well as technical assistance in the days before final manuscript submission. We thank the authors for their effort to write and rewrite chapters so as to improve the overall quality and integration of the book, for their patience and cooperation. We are particularly grateful to Cambridge University Press, and namely Alan Crowden, Clare Georgy and Dominic Lewis, for their assistance with the production of this volume.

\section{References}

Allen, A. P., Brown, J. H. \& Gillooly, J. F. (2002). Global biodiversity, biochemical kinetics, and the energetic-equivalence rule. Science, 297, 1545-1548.

Arrhenius, O. (1921). Species and area. Journal of Ecology, 9, 95-99.
Blackburn, T. M. \& Gaston, K. J. (eds.) (2003). Macroecology: Concepts and Consequences. Oxford: British Ecological Society and Blackwell Science.

Bonn, A., Storch, D. \& Gaston, K. J. (2004). Structure of the species-energy 


\section{Cambridge University Press}

978-0-521-87602-5 - Scaling Biodiversity

Edited by David Storch, Pablo A. Marquet and James H. Brown

Excerpt

More information

relationship. Proceedings of the Royal Society of London, Series B, 271, 1685-1691.

Brown, J.H., Gillooly, J. F., Allen, A. P., Savage, V. M. \& West, G. B. (2004). Toward a metabolic theory of ecology. Ecology, 85, 1771-1789.

Currie, D. J., Mittelbach, G. G., Cornell, H. V., et al. (2004). Predictions and tests of climatebased hypotheses of broad-scale variation in taxonomic richness. Ecology Letters, 7, 1121-1134.

Fenchel, T. \& Finlay, B. J. (2004). The ubiquity of small species: patterns of local and global diversity. Bioscience, 54, 777-784.

Gaston, K. J. (2000). Global patterns in biodiversity. Nature, 405, 220-227.

Gaston, K. J. \& Blackburn, T. M. (2000). Pattern and Process in Macroecology. Oxford: Blackwell Science.

Gleason, H. A. (1922). On the relation between species and area. Ecology, 3, 158-162.

Hanski, I. \& Gyllenberg, M. (1997). Uniting two general patterns in the distribution of species. Science, 275, 397-400.

Harte, J., Kinzig, A. \& Green, J. (1999). Selfsimilarity in the distribution and abundance of species. Science, 284, 334-336.

Harte, J., Conlisk, E., Ostling, A., Green, J. L. \& Smith, A. B. (2005). A theory of spatial structure in ecological communities at multiple spatial scales. Ecological Monographs, 75, 179-197.

Hawkins, B. A., Field, R., Cornell, H. V., et al. (2003). Energy, water, and broad-scale geographic patterns of species richness. Ecology, 84, 3105-3117.

He, F. L. \& Legendre, P. (2002). Species diversity patterns derived from species-area models. Ecology, 85, 1185-1198.

Hubbell, S. P. (2001). The Unified Theory of Biodiversity and Biogeography. Princeton: Princeton University Press.

Keitt, T. H. \& Stanley, H. E. (1998). Dynamics of North American breeding bird populations. Nature, 393, 257-260.
Keitt, T. H., Amaral, L. A. N., Buldryev, S. V. \& Stanley, H. E. (2002). Scaling in the growth of geographically subdivided populations: invariant patterns from a continent-wide biological survey. Philosophical Transactions of the Royal Society of London, Series B, 357, 627-633.

Koleff, P., Gaston K. J. \& Lennon, J. J. (2003). Measuring beta diversity for presenceabsence data. Journal of Animal Ecology, 72, 367-382.

Kunin, W.E. (1998). Extrapolating species abundances across spatial scales. Science, 281, 1513-1515.

Maurer, B. A. (1999). Untangling Ecological Complexity? The Macroscopic Perspective. Chicago: University of Chicago Press.

Preston, F. W. (1960). Time and space and the variation of species. Ecology, 29, 254-283.

Rahbek, C. (2005). The role of spatial scale and the perception of large-scale species-richness patterns. Ecology Letters, 8, 224-239.

Rahbek, C. \& Graves, G. R. (2001). Multiscale assessment of patterns of avian species richness. Proceedings of the National Academy of Sciences of the United States of America, 98, 4534-4539.

Rosenzweig, M. L. (1995). Species Diversity in Space and Time. Cambridge: Cambridge University Press.

Šizling, A. L. \& Storch, D. (2004). Power-law species-area relationships and self-similar species distributions within finite areas. Ecology Letters, 7, 60-68.

Storch, D. \& Gaston, K. J. (2004). Untangling ecological complexity on different scales of space and time. Basic and Applied Ecology, 5, 389-400.

Storch, D., Šizling, A. L. \& Gaston, K. J. (2003). Geometry of the species-area relationship in central European birds: testing the mechanism. Journal of Animal Ecology, 72, 509-519. 